\title{
Premature mortality in Belgium in 1993-2009: leading causes, regional disparities and 15 years change
}

\author{
Françoise Renard ${ }^{1 *}$, Jean Tafforeau ${ }^{1}$ and Patrick Deboosere ${ }^{2}$
}

\begin{abstract}
Background: Reducing premature mortality is a crucial public health objective. After a long gap in the publication of Belgian mortality statistics, this paper presents the leading causes and the regional disparities in premature mortality in 2008-2009 and the changes since 1993.

Methods: All deaths occurring in the periods 1993-1999 and 2003-2009, in people aged 1-74 residing in Belgium were included.

The cause of death and population data for Belgium were provided by Statistics Belgium, while data for international comparisons were extracted from the WHO mortality database.

Age-adjusted mortality rates and Person Year of Life Lost (PYLL) were calculated. The Rate Ratios were computed for regional and international comparisons, using the region or country with the lowest rate as reference; statistical significance was tested assuming a Poisson distribution of the number of deaths.

Results: The burden of premature mortality is much higher in men than in women (respectively $42 \%$ and $24 \%$ of the total number of deaths). The 2008-9 burden of premature mortality in Belgium reaches 6410 and 3440 PYLL per 100,000, respectively in males and females, ranking 4th and 3rd worst within the EU15. The disparities between Belgian regions are substantial: for overall premature mortality, respective excess of 40\% and 20\% among males, 30\% and 20\% among females are observed in Wallonia and Brussels as compared to Flanders. Also in cause specific mortality, Wallonia experiences a clear disadvantage compared to Flanders. Brussels shows an intermediate level for natural causes, but ranks differently for external causes, with less road accidents and suicide and more non-transport accidents than in the other regions.

Age-adjusted premature mortality rates decreased by $29 \%$ among men and by $22 \%$ among women over a period of 15 years. Among men, circulatory diseases death rates decreased the fastest $(-43.4 \%)$, followed by the neoplasms $(-26.6 \%)$, the other natural causes $(-21.0 \%)$ and the external causes $(-20.8 \%)$. The larger decrease in single cause is observed for stomach cancer $(-48.4 \%)$, road accident $(-44 \%)$, genital organs $(-40.4 \%)$ and lung $(-34.6 \%)$ cancers. On the opposite, liver cancer death rate increased by $16 \%$.

Among female, the most remarkable feature is the $50.2 \%$ increase in the lung cancer death rate. For most other causes, the decline is slightly weaker than in men.

Conclusion: Despite a steady decrease over time, international comparisons of the premature mortality burden highlight the room for improvement in Belgium. The disadvantage in Wallonia and to some extent in Brussels suggest the role of socio-economic factors; well- designed health policies could contribute to reduce the regional disparities.

The increase in female lung cancer mortality is worrying.
\end{abstract}

Keywords: Premature mortality, Mortality rates, Potential Years of Life Lost, Causes of death, Belgium

\footnotetext{
* Correspondence: francoise.renard@wiv-isp.be

'Department Public Health and Statistics, Institute of Public Health, Brussels, Belgium

Full list of author information is available at the end of the article
}

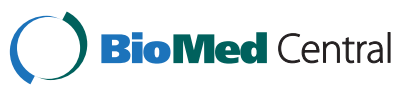

(c) 2014 Renard et al.; licensee BioMed Central Ltd. This is an Open Access article distributed under the terms of the Creative Commons Attribution License (http://creativecommons.org/licenses/by/4.0), which permits unrestricted use, distribution, and reproduction in any medium, provided the original work is properly credited. The Creative Commons Public Domain Dedication waiver (http://creativecommons.org/publicdomain/zero/1.0/) applies to the data made available in this article, unless otherwise stated. 


\section{Background}

Reducing premature deaths is a crucial goal of public health policies [1]; premature mortality statistics are invaluable indicators to assist important public health functions: the establishment of priorities, the monitoring of temporal trends to evaluate the impact of public health actions and the detection of geographical disparities that can highlight problems in resources allocations or non-medical health determinants $[2,3]$.

Traditional age-adjusted mortality rates allow for temporal or geographical comparisons of premature death figures. However, as stated by the US Centre for Diseases Control and Prevention (CDC) [2], death rates often fail to tell the entire story of premature mortality: since most deaths occur in older age-groups, mortality rates are dominated by the underlying diseases of the elderly. An alternative measure has been proposed $[2,4,5]$, the "Potential Years of Life Lost (PYLL)", an indicator weighting the deaths occurring at younger ages more heavily than those occurring later. It has been largely used as a planning tool since the 1980s.

In Belgium, previous studies analysed the cause-specific (premature) mortality either using rates or PYLL [6-9]. The latest published studies refer to years up to 1997; afterwards, there has been a long hiatus in the publication of mortality data due to a reorganisation of the responsibilities in processing the death certificates. For more than 12 years, Belgian mortality data were only partially available on the regional levels. Recently, causes of deaths data have been produced again at the national level. Those are currently (December 2013 ) available for nearly all years previous to 2009 (except for 2000-2002). This prompted us to analyse the recent patterns of premature mortality. The current paper presents the 2008-9 premature mortality figures using both death rates and PYLL; the aim is to identify the leading causes of premature death, to look at regional disparities and to monitor the changes since 1993. In addition the overall Belgian premature mortality level is compared to the EU15 countries.

\section{Methods}

\section{Period and people under study}

Premature mortality is defined here as death occurring before 75 years. All deaths occurring in the periods 1993-1999 and 2003-2009 (last available years at national level in December 2013), in people aged 1 to 74 years and residing in Belgium were included. Infant deaths are excluded from the study.

Cause of death and population data for Belgium are provided by Statistics Belgium, Directorate in charge of the production of vital statistics at the national level. The causes of death are specified in deaths certificates by a medical doctor; they are subsequently coded according to the ICD rules by trained staff within 2 regional Health Agencies (one for Flanders and Brussels, one for Wallonia), before being pooled at the national level by Statistics Belgium. Regular coordination meetings between the regional and federal levels are organised in order to guarantee consistency in the coding/registration rules.

Deaths and population data for International comparisons: deaths and population data by sex and 5 years age groups for the EU15 countries were extracted from the WHO Mortality Database to compare overall PYLL rates.

Classifications in use: the 9th revision of the International Classification of Disease (ICD-9) was in use until 1997 while the ICD-10 was introduced in $1998[10,11]$. Causes of deaths were poorly coded (except for neoplasms) in the first 2 years after the adoption of the 10th revision (1998-1999); consequently for those 2 years only overall and cancer mortality will be published. The transition in ICD-classification created also jumps in the subclasses of circulatory diseases; hence those will not be used in the trends analysis. As suicide is often suspected being underreported, several correction methods have been proposed [12-15]. We will, in addition to the registered suicide data, calculate estimations by using Jougla's method [13].

Grouping of the causes of death: the causes of death are displayed according to the ICD-chapters. Chapters with less than 200 yearly cases for Belgium are not shown separately. Besides, single causes or group of causes are presented for cancers, circulatory diseases and external causes, as well as for some single causes also relevant for health promotion (chronic liver diseases, COPD). The classification used along with ICD-9 and ICD-10 is summarized in Table 1.

Indicators: age-standardised death rates (1-74 year) are computed with the direct method [16]. Potential Years of Life Lost (PYLL), at population level, between 1 and 74 years are computed as the summation of the remaining years, had the deceased lived up to 75 years. Individual age at death was used for the computation. The age adjusted PYLL rates per 100.000 are calculated according to the method described by Romeder [5]. Mortality rates and PYLL-rates used for Belgian regional or time-trends comparisons are age-adjusted with the 2000 Belgian population as a standard, while for EU15 comparisons, the European Standard population [17] is used.

\section{Comparisons and statistical analysis}

- Cause specific mortality rates were compared between the regions and between EU15 countries with the Rate Ratios of the age-adjusted mortality rates, using the region/country with the lowest rate as reference (Flanders, Sweden Spain). To test for 
Table 1 ICD-9 and ICD10 codes for the categories and single causes of deaths used

\begin{tabular}{|c|c|c|c|c|c|}
\hline & ICD chapter & $\begin{array}{l}\text { Selected single causes } \\
\text { or group of causes }\end{array}$ & ICD9 & ICD10 & $\begin{array}{l}\text { Clusters of main causes } \\
\text { (used in Table 2) }\end{array}$ \\
\hline \multirow[t]{26}{*}{$\begin{array}{l}\text { Natural } \\
\text { causes }\end{array}$} & $\begin{array}{l}\text { Inf.\&parasit. } \\
\text { diseases }\end{array}$ & & $1-139$ & A00-B99 & \\
\hline & Neoplasms & & $140-239$ & C00-D48 & Neoplasms \\
\hline & & Head,neck\& oesoph.Ca & $140-149 ; 161 ; 150$ & $\mathrm{C} 00-\mathrm{C} 14 ; \mathrm{C} 30-\mathrm{C} 32 ; \mathrm{C} 15$ & \\
\hline & & Stomach Ca & 151 & $\mathrm{C} 16$ & \\
\hline & & Colorectal Ca & $153 ; 154.0 ; 154.1$ & C18-C20 & \\
\hline & & Liver Ca & 155 & $\mathrm{C} 22$ & \\
\hline & & Pancreas Ca & 157 & $\mathrm{C} 25$ & \\
\hline & & Lung Ca & 162 & C33-C34 & \\
\hline & & Female Breast $\mathrm{Ca}$ & 174 & C50 & \\
\hline & & Female genital org.Ca & $179-184$ & C51-C58 & \\
\hline & & Male genital org.Ca & $185-187$ & C60-C63 & \\
\hline & & CNS Ca & 191-192 & $\mathrm{C} 70-\mathrm{C} 72$ & \\
\hline & & Hematol.Ca & $200-208$ & C81-C96 & \\
\hline & & Other Neoplasms & & & \\
\hline & Endocrine dis & & $240-279$ & E00-E99 & \\
\hline & $\begin{array}{l}\text { Mental\&Neurol } \\
\text { Dis. }\end{array}$ & & 290-319; 320-389 & F00-F99; G00-G99 & \\
\hline & $\begin{array}{l}\text { Circulatory } \\
\text { diseases }\end{array}$ & & $390-459$ & $100-199$ & Circulatory diseases \\
\hline & & Isc.Heart Dis. & & & \\
\hline & & Cerebrov.dis.\&HTA & & & \\
\hline & & Other Circ.Dis. & & & \\
\hline & Resp.Syst.dis. & & $460-519$ & J00-J99 & All Other natural causes \\
\hline & & Chron.Obs.Pulm.Dis. & & & \\
\hline & Digest.Syst.Dis. & & $520-579$ & K00-K93 & \\
\hline & & Chronic Liver Dis. & & & \\
\hline & $\begin{array}{l}\text { Genito-urin. } \\
\text { Syst.Dis }\end{array}$ & & $580-629$ & No0-N99 & \\
\hline & Sympt.\&lll-def. & & 780-799 & R00-R99 & \\
\hline \multirow{5}{*}{$\begin{array}{l}\text { External } \\
\text { causes }\end{array}$} & External causes & & E800-899 & V01-Y98 & External causes \\
\hline & & Road Accident & E810-829 & V01-V80;V82-V89,V99 & \\
\hline & & Non transp.acc.(Poison/fall/envir.) & $\begin{array}{c}\text { E850-859;E880-888; } \\
\text { E890-929 }\end{array}$ & X00-X49;W00-W99; & \\
\hline & & Suicide & E950-959 & X60-X84 & \\
\hline & & $\begin{array}{l}\text { Event of Undet.Intent Miscell. other } \\
\text { external causes** }\end{array}$ & $\begin{array}{l}\text { E980-989 Remaining } \\
\text { ext. codes }\end{array}$ & $\begin{array}{l}\text { Y10-Y34 Remaining } \\
\text { ext. codes }\end{array}$ & \\
\hline
\end{tabular}

${ }^{*}$ Chapters with less than 200 yearly cases were not displayed.

**Miscellaneous other external causes include non-road transport accidents (train, plane, water), homicide, iatrogenic, legal interventions and war.

the statistical significance, we first calculated the variance of the age-adjusted rates using a Poisson approximation as $[16,18]$ :

$\operatorname{Var}(A S R)=\sum\left(a_{i} w_{i}^{2} * 100.000 / n_{i}\right) /\left(\sum w_{i}\right)^{2}$
A z-test [19] was then performed as $z=(A S R 1-A S R 2) /$ $S Q R T(\operatorname{Var}(A S R 1)+\operatorname{Var}(A S R 2))$

The $95 \%$ confidence interval of the age-adjusted rates was also computed for the international rates.

Where ASR = Age Standardized rates; $a_{i}=$ age- specific rates per 100.000 in the age-group $i ; n_{i}=$ effectif of the population for the age group $\mathrm{i} ; \mathrm{w}_{\mathrm{i}}=$ effectif of the 
standard population for the age group i, $\operatorname{var}(\mathrm{ASR})=$ variance of the age-standardized rates.

Beside the statistical comparison, the EU countries were ranked by sex based on the burden of overall premature mortality expressed in PYLL; the causes of deaths were also ranked by region in function of their burden expressed in PYLL.

The evolution between the 1st and 4th period is expressed as a relative rate difference (Rate 4th -Rate $1 \mathrm{st}) /$ rate 1st; the statistical significance was tested with a $\mathrm{z}$-test calculated the same way as for regional comparisons.

All analyses were performed using SAS 9.3 statistical software.

\section{Results}

Overall premature mortality and share of the main categories of causes of death in 2008-9

Among the 207.571 deaths that occurred in Belgium in 2008-2009, we focused here on the 69.571 deaths (33.5\% of the total) that were non-infant premature deaths $(1-74 \mathrm{yr})$; the remaining deaths were infant deaths $(0.4 \%$ of all deaths), and deaths occurring in people aged 75 and over (66.1\%). While the total number of deaths is similar in both sexes, the fraction of those deaths that occurs prematurely (as defined here before 75) is much higher in men than in women, representing $42 \%$ and $24 \%$ of the total deaths respectively.

Belgium ranks 4th worst for premature mortality in males and 3rd worst for females in the EU15 (ranked on PYLL) (Table 2) with a quite similar ranking based on rates. Compared to the countries with the lowest rates (Sweden and Spain, respectively for men and women) the premature mortality Rate Ratios for Belgium are 1.31 in men and 1.40 in women.

Table 3 displays the age-adjusted death rates (1-74 years), PYLL and share by sex for the main categories of conditions (neoplasms, circulatory diseases, all other natural causes, and external causes) in 2008-9. Male to female ratio is nearly 2 for the overall mortality rates/PYLL, but varies with the specific causes, ranging from 1.6 and 1.3 (as expressed respectively in rates or in PYLL) for neoplasms to 2.6 and 3.0 for external causes.

Neoplasms represent the first group of premature mortality, both in men and women. The next cause depends on the indicator selected: using mortality rates, the mixed group of "all other natural causes" ranks second, followed by circulatory diseases and external causes, the latter representing $13.2 \%$ and $9.5 \%$ in males and females respectively. However, when using PYLL, external causes represent respectively $28.0 \%$ and $17.7 \%$ in males and females. This shift is due to the fact that most external causes of death occur in younger people.
Regional differences in overall and cause-specific premature deaths

Important regional differences are observed both in overall and in cause-specific premature mortality (Tables 4 and 5). Flanders has the lowest overall premature deaths rates in both sexes (409.6 per 100.000 in males and 224.4 per 100.000 in females). Overall rates in Brussels and Wallonia are respectively 20 and $40 \%$ higher in men and 20 and $30 \%$ higher in women compared to Flanders.

In men, important regional differences are also observed for specific causes: compared to Flanders, Wallonia experiences a $20 \%$ excess in cancer death rates (rates ratio $=1.2$ ). Higher death rates are observed in head \& neck, lung, liver, pancreas and the miscellaneous other neoplasms, while no regional difference was observed in stomach, colorectal, reproductive organs, haematological and central nervous system cancers. Circulatory diseases present an excess of 30\% in Wallonia. The highest excess of death rates $(80 \%)$ is seen for the miscellaneous group of 'All other natural causes', in which the excess ranges between 70 and $110 \%$, depending on the specific causes. The group of external causes presents a $50 \%$ excess in Wallonia, with $60 \%$ in road as well as in non-transport accidents and $40 \%$ for reported suicide.

Compared to Flanders, Brussels presents a 10\% excess in male cancer premature death rates (1-74 years). Only liver cancer and "miscellaneous other cancer" show a statistically significant excess with respect to Flanders. Deaths from circulatory diseases and all other natural causes are respectively $20 \%$ and $60 \%$ higher than in Flanders. Among the external causes, death rates from road accidents in Brussels represent half the Flemish rates, while the rates for non-transport accidents is 50\% higher. Reported suicide rates are 20\% lower than in Flanders. The death rate for external events of undetermined intent is much higher in Brussels than in the other regions.

In women, excess in Brussels and Walloon's rates are observed for the same natural causes of death as for men, with a slightly smaller magnitude, except for head \& neck and lung cancer death rates with a $40 \%$ excess over Flanders rates in women (against 20\% in men). There is no regional difference in the premature breast, gynaecological, colorectal, haematological and CNS cancer death rate. Among external causes, suicide rate in women is $18 \%$ higher in Wallonia than in Flanders (versus $40 \%$ higher in men).

\section{Reported and estimated suicide rates}

As the categories "event of undetermined intent" (Y10-Y34) and ill-defined and unknown conditions (R96-R99) potentially include suicides, Jougla's correction [13] has been applied to the reported suicide rates; estimated male suicide rates $(1-74 \mathrm{yr})$ respectively in 
Table 2 Premature mortality (1-75 yr) in EU-15 countries, 2008-2009 Person-years of Life Lost, Rates and Ranking

\begin{tabular}{|c|c|c|c|c|c|c|}
\hline Country & PYLL 1-75 & RANK Pyll 1-75 & RATES 1-75 & RANK Rates 1-75 & RATIO Rates 1-75 & 95\% Cl Rates 1-75 \\
\hline \multicolumn{7}{|c|}{ Sex $=$ Males } \\
\hline Finland & 7177 & 1 & 437.6 & 1 & 1.45 & {$[432.1 ; 443.0]$} \\
\hline Portugal & 6997 & 2 & 428.9 & 3 & 1.42 & {$[425.0 ; 432.8]$} \\
\hline France & 6477 & 3 & 392.1 & 5 & 1.30 & {$[390.5 ; 393.7]$} \\
\hline Belgium & 6211 & 4 & 396.5 & 4 & 1.31 & {$[392.7 ; 400.2]$} \\
\hline Greece & 6183 & 5 & 379.6 & 7 & 1.26 & {$[376.1 ; 383.1]$} \\
\hline Denmark & 6151 & 6 & 429.5 & 2 & 1.42 & {$[424.1 ; 434.9]$} \\
\hline Austria & 5817 & 7 & 378.9 & 8 & 1.25 & [374.8; 383.0] \\
\hline Ireland & 5798 & 8 & 372.1 & 9 & 1.23 & {$[366.0 ; 378.3]$} \\
\hline Germany & 5751 & 9 & 388.3 & 6 & 1.29 & [387.0; 389.6] \\
\hline United Kingdom & 5628 & 10 & 362.6 & 10 & 1.20 & {$[361.1 ; 364.1]$} \\
\hline Spain & 5476 & 11 & 358.9 & 11 & 1.19 & {$[357.2 ; 360.7]$} \\
\hline Luxembourg & 4862 & 12 & 330.5 & 12 & 1.09 & {$[314.1 ; 347.0]$} \\
\hline Italy & 4727 & 13 & 314.2 & 14 & 1.04 & {$[312.9 ; 315.6]$} \\
\hline Netherlands & 4544 & 14 & 326.0 & 13 & 1.08 & {$[323.3 ; 328.7]$} \\
\hline Sweden & 4518 & 15 & 302.1 & 15 & 1.00 & {$[298.7 ; 305.5]$} \\
\hline EU15 mean & 5661 & . & 367.8 & . & . & {$[367.2 ; 368.4]$} \\
\hline \multicolumn{7}{|c|}{ Sex $=$ Females } \\
\hline Country & PYLL 1-75 & RANK Pyll 1-75 & RATES 1-75 & RANK Rates 1-75 & RATIO Rates 1-75 & 95\% Cl Rates 1-75 \\
\hline Denmark & 3724 & 1 & 272.0 & 1 & 1.78 & {$[267.8 ; 276.2]$} \\
\hline United Kingdom & 3403 & 2 & 227.8 & 2 & 1.50 & {$[226.7 ; 229.0]$} \\
\hline Belgium & 3341 & 3 & 213.9 & 4 & 1.40 & {$[211.2 ; 216.5]$} \\
\hline Ireland & 3259 & 4 & 216.5 & 3 & 1.42 & {$[211.9 ; 221.2]$} \\
\hline Netherlands & 3137 & 5 & 211.0 & 5 & 1.38 & {$[208.8 ; 213.1]$} \\
\hline Finland & 3086 & 6 & 194.3 & 7 & 1.27 & [190.8; 197.8] \\
\hline Portugal & 3063 & 7 & 192.9 & 8 & 1.27 & [190.4; 195.3] \\
\hline Germany & 3008 & 8 & 201.5 & 6 & 1.32 & [200.6; 202.4] \\
\hline France & 2953 & 9 & 177.3 & 12 & 1.16 & {$[176.3 ; 178.4]$} \\
\hline Austria & 2861 & 10 & 188.9 & 10 & 1.24 & {$[186.1 ; 191.7]$} \\
\hline Sweden & 2736 & 11 & 189.8 & 9 & 1.25 & {$[187.1 ; 192.4]$} \\
\hline Greece & 2544 & 12 & 164.5 & 13 & 1.08 & {$[162.3 ; 166.7]$} \\
\hline Luxembourg & 2514 & 13 & 182.5 & 11 & 1.20 & {$[170.5 ; 194.5]$} \\
\hline Italy & 2425 & 14 & 162.8 & 14 & 1.07 & {$[161.9 ; 163.7]$} \\
\hline Spain & 2422 & 15 & 152.4 & 15 & 1.00 & [151.3; 153.5] \\
\hline EU15 mean & 2912 & . & 190.2 & . & & [189.8; 190.6] \\
\hline
\end{tabular}

Rates and Pyll are expressed per 100.000 Person-Years and age-standardized on European population Author's own calculation.

Flanders, Brussels and Wallonia are 26.1, 24.2 and 36.0 per 100.000 and estimated Potential Years of Life Lost are 763, 607 and 997 per 100.000. Compared to the registered rates (Tables 4 and 5), this represents an increase of $10 \%$ in Flanders, $31 \%$ in Brussels and $8 \%$ in Wallonia.

In women, the estimated suicide rates (1-74 year) after Jougla's correction are 10.4, 10.6 and 12.3 per 100.000, and the estimated Potential years of Life Lost are 283,
263 and 316 per 100.000, respectively in Flanders, Brussels and Wallonia. Compared to the registered rates (Tables 4 and 5), this represents an increase of $11 \%$ in Flanders, 34\% in Brussels and 12\% in Wallonia.

\section{Ranking the top 20 causes of deaths.}

Tables 6 and 7 ranks the causes of deaths according to PYLL in each region, for 2008-2009. It is noteworthy 
Table 3 Major groups of premature death (1-74 yr), Belgium 2008-2009 Age-adjusted rates and Potential Years of Life Lost

\begin{tabular}{|c|c|c|c|c|c|c|c|c|c|c|}
\hline & \multicolumn{4}{|l|}{ Males } & \multicolumn{4}{|l|}{ Females } & \multicolumn{2}{|c|}{ Sex ratio } \\
\hline & Age-adj. RATES & $\%$ & Age-adj.PYLL & $\%$ & Age-adj. RATES & $\%$ & Age-adj.PYLL & $\%$ & RATES & PYLL \\
\hline NEOPLASMS & 175.2 & 37.6 & 1914.1 & 29.9 & 111 & 44.2 & 1444.9 & 42.0 & 1.6 & 1.3 \\
\hline OTH. NAT. CAUSES* & 120 & 25.7 & 1524.9 & 23.8 & 67.5 & 26.9 & 879.2 & 25.6 & 1.8 & 1.7 \\
\hline CIRCUL. DISEASES & 109.6 & 23.5 & 1176.2 & 18.4 & 49 & 19.5 & 509.6 & 14.8 & 2.2 & 2.3 \\
\hline EXTERNAL CAUSES & 61.5 & 13.2 & 1795.3 & 28.0 & 23.8 & 9.5 & 605.9 & 17.6 & 2.6 & 3.0 \\
\hline TOTAL & 466.3 & 100.0 & 6410.4 & 100.0 & 251.2 & 100.0 & 3439.6 & 100.0 & 1.9 & 1.9 \\
\hline
\end{tabular}

*Oth. Nat. causes = all natural causes except Neoplasms and Circulatory Diseases.

that the ranking would be different based on the indicator used: external causes (suicide, road accident, other accidents) are ranking higher when expressed in PYLL than in rates, while natural causes of deaths (lung cancer, IHD, other circulatory diseases) rank lower. For instance, the suicide rate in men is much lower than the lung cancer rates (respectively 26 and 62 per 100.000), meaning that about 3 times as many men are dying from lung cancer as from suicide. However, in terms of Potential Years of Life lost, suicide ranks first, due to the fact that a great part of the suicides occur in younger people than lung cancer deaths.

In men, at Belgian level, suicide, lung cancer, road accident, ischaemic heart diseases and other circulatory diseases deaths rank highest if expressed in PYLL, while suicide and road accidents rank respectively 5th and 12th if expressed in mortality rates.

The ranking is quite similar for Flanders and Wallonia, but differs in Brussels with respect to external causes: reported suicide ranks 3rd and road accident 12th (expressed in PYLL). However, as events of undetermined intent rank much higher in Brussels, an estimated suicide figure was computed, that ranks $2 \mathrm{~d}$ in Brussels (instead of 3rd) when expressed in PYLL and 6th (instead of 9th) when expressed in rates.

In women, at Belgium level, breast cancer is the leading cause of premature death (expressed in PYLL as well as in rates). It is followed by suicide, lung cancer, other circulatory diseases, diseases of the digestive system. Breast cancer is also the leading cause of death in all 3 regions; it is followed by suicide in Flanders, and by lung cancer in Brussels and Wallonia, where suicide respectively occupies the 4th and 3rd place (ranked on PYLL). After correction, the estimated suicide figure in women ranks 3rd in Brussels (instead of 4th) when expressed in PYLL, and 11th (instead of 15th) if expressed in mortality rates.

\section{Leading causes by age-group}

Tables 8 and 9 details the 5 leading causes of deaths according to age-specific mortality rates within broad agegroups by region.
Under the age of 40, suicide and accidents rank first in all regions and in both sexes.

Lung cancers and ischemic heart diseases are the main causes in men after 40, while suicide progressively occupies a lower place.

Between 40 and 59 years, breast and lung cancer deaths occupy the first places in women in all regions. In women aged 60-74, the ranking and the specific rates differ considerably between regions: breast cancer ranks first only in Flanders (in term of mortality rates), with slightly higher rates than in the other regions. The other circulatory and respiratory diseases rank first in Wallonia, with much higher mortality rates than in Flanders. Lung cancer deaths mortality rates are considerably higher in Brussels (where it ranks first) and Wallonia than in Flanders.

\section{Evolution over time}

Tables 10 and 11 shows the evolution of the overall and cause specific age-standardised death rates (1-74 years) and PYLL across 4 time periods (separated by 5 years): 1993-1994, 1998-1999, 2003-2004 and 2008-2009. For the period 1998-1999, only overall and cancer death rates are displayed because of a lack of reliability for the other causes of death.

Overall premature death rates in men steadily decreased over the 4 time periods (Tables 10 and 11, 5th column), reaching a $29.6 \%$ decrease between the first (1993-4) and the 4th period (2008-2009). Mortality rates resulting of natural causes decreased faster $(-30.7 \%)$ than for external causes $(-20.8 \%)$. Within the natural causes, circulatory diseases death rates decreased the fastest $(-43.4 \%)$, followed by the neoplasms $(-26.6 \%)$ and the other natural causes $(-21.0 \%)$. The decrease in specific cancer death rates greatly differs by organ: change was the highest for stomach $(-48.4 \%)$, followed by genital organs $(-40.4 \%)$ and lung $(-34.6 \%)$ cancers. Colorectal, head \& neck and CNS cancers decrease by more than 20\%. Pancreas cancer death rates remain stable. Finally, an increase was observed for liver cancer $(+16 \%)$. 
Table 4 Regional Comparisons of Causes of Premature deaths in Males, Belgium 2008-2009

\begin{tabular}{|c|c|c|c|c|c|c|c|c|c|c|c|}
\hline $\begin{array}{l}\text { Causes of death } \\
\text { grouped }\end{array}$ & Detailed cause & $\begin{array}{c}\text { RATES } \\
\text { Belgium }\end{array}$ & $\begin{array}{c}\text { PYLL } \\
\text { Belgium }\end{array}$ & $\begin{array}{c}\text { RATES } \\
\text { Flanders }\end{array}$ & $\begin{array}{c}\text { PYLL } \\
\text { Flanders }\end{array}$ & $\begin{array}{c}\text { RATES } \\
\text { Brussels }\end{array}$ & $\begin{array}{c}\text { PYLL } \\
\text { Brussels }\end{array}$ & $\begin{array}{l}\text { Rates ratio } \\
\text { Bxl/Fla }\end{array}$ & $\begin{array}{c}\text { RATES } \\
\text { Wallonia }\end{array}$ & $\begin{array}{c}\text { PYLL } \\
\text { Wallonia }\end{array}$ & $\begin{array}{l}\text { Rates ratio } \\
\text { Wal/Fla }\end{array}$ \\
\hline ALL PREMATURE & & 466.3 & 6410 & 409.6 & 5491 & 492.9 & 6521 & $1.2^{* *}$ & 572.2 & 8161 & $1.4^{* *}$ \\
\hline $\begin{array}{l}\text { NATURAL } \\
\text { CAUSES }\end{array}$ & & 404.7 & 4615 & 356.0 & 3883 & 436.8 & 5070 & $1.2^{* *}$ & 493.2 & 5894 & $1.4^{* *}$ \\
\hline \multirow{11}{*}{$\begin{array}{l}\text { ALL } \\
\text { NEOPLASMS }\end{array}$} & & 175.1 & 1914 & 164.0 & 1735 & 176.4 & 2069 & $1.1^{*}$ & 196.4 & 2210 & $1.2^{* *}$ \\
\hline & $\begin{array}{l}\text { Head, Neck \& } \\
\text { oesoph.Ca }\end{array}$ & 16.6 & 219 & 15.6 & 199 & 17.0 & 220 & 1.1 & 18.6 & 258 & $1.2^{* *}$ \\
\hline & Stomach Ca & 5.1 & 56 & 4.9 & 52 & 6.6 & 95 & 1.3 & 5.0 & 53 & 1.0 \\
\hline & Colorectal Ca & 15.6 & 152 & 15.2 & 143 & 15.8 & 169 & 1.0 & 16.3 & 163 & 1.1 \\
\hline & Liver Ca & 5.9 & 64 & 4.8 & 53 & 7.7 & 89 & $1.6^{*}$ & 7.6 & 80 & $1.6^{* *}$ \\
\hline & Pancreas Ca & 9.0 & 92 & 8.3 & 83 & 10.3 & 105 & 1.2 & 10.0 & 107 & $1.2^{*}$ \\
\hline & Lung Ca & 61.9 & 630 & 58.2 & 564 & 56.8 & 625 & 1.0 & 70.1 & 754 & $1.2^{* *}$ \\
\hline & Male genital org.Ca & 8.8 & 65 & 8.4 & 61 & 8.5 & 64 & 1.0 & 9.6 & 73 & 1.1 \\
\hline & CNS Ca & 5.9 & 106 & 6.3 & 111 & 4.9 & 86 & 0.8 & 5.4 & 104 & 0.9 \\
\hline & Hematol.Ca & 11.5 & 138 & 11.3 & 135 & 10.7 & 155 & 1.0 & 12.2 & 141 & 1.1 \\
\hline & Other Neoplasms & 34.9 & 391 & 31.0 & 336 & 38.0 & 462 & $1.2^{*}$ & 41.6 & 477 & $1.3^{* *}$ \\
\hline \multirow{4}{*}{$\begin{array}{l}\text { CIRCULATORY } \\
\text { DISEASES }\end{array}$} & & 109.6 & 1176 & 99.3 & 1032 & 115.6 & 1257 & $1.2^{* *}$ & 129.0 & 1434 & $1.3^{* *}$ \\
\hline & Isc.Heart Dis. & 47.6 & 512 & 42.1 & 431 & 53.6 & 571 & $1.3^{* *}$ & 57.0 & 656 & $1.4^{* *}$ \\
\hline & Cerebrov.dis.\&HTA & 20.7 & 209 & 18.4 & 185 & 21.5 & 239 & 1.2 & 25.3 & 249 & $1.4^{* *}$ \\
\hline & Other Circ.Dis. & 41.3 & 455 & 38.8 & 416 & 40.4 & 447 & 1.0 & 46.7 & 529 & $1.2^{* *}$ \\
\hline $\begin{array}{l}\text { ALL OTH.NAT. } \\
\text { CAUSES }\end{array}$ & & 120.0 & 1525 & 92.8 & 1116 & 144.8 & 1743 & $1.6^{* *}$ & 167.9 & 2250 & $1.8^{* *}$ \\
\hline $\begin{array}{l}\text { Inf.\&parasit. } \\
\text { diseases }\end{array}$ & & 8.6 & 116 & 6.1 & 79 & 13.1 & 187 & $2.1^{* *}$ & 12.6 & 166 & $2.1^{* *}$ \\
\hline Endocrine dis & & 9.1 & 122 & 6.6 & 98 & 13.2 & 163 & $2.0^{* *}$ & 13.3 & 160 & $2.0^{* *}$ \\
\hline $\begin{array}{l}\text { Mental\&Neurol } \\
\text { Dis. }\end{array}$ & & 22.7 & 345 & 18.3 & 249 & 21.8 & 276 & 1.2 & 31.7 & 546 & $1.7^{* *}$ \\
\hline \multirow[t]{2}{*}{ Resp.Syst.dis. } & & 36.3 & 315 & 29.1 & 237 & 43.8 & 370 & $1.5^{* *}$ & 48.9 & 451 & $1.7^{* *}$ \\
\hline & Chron.Obs.Pulm.Dis. & 21.5 & 170 & 16.8 & 122 & 25.7 & 200 & $1.5^{* *}$ & 30.0 & 254 & $1.8^{* *}$ \\
\hline \multirow[t]{2}{*}{ Digest.Syst.Dis. } & & 24.8 & 356 & 19.1 & 250 & 28.5 & 436 & $1.5^{* *}$ & 34.9 & 534 & $1.8^{* *}$ \\
\hline & Chronic Liver Dis. & 13.5 & 217 & 9.8 & 145 & 14.8 & 253 & $1.5^{* *}$ & 20.2 & 342 & $2.1^{* *}$ \\
\hline $\begin{array}{l}\text { Genito-urin.Syst. } \\
\text { Dis }\end{array}$ & & 4.0 & 34 & 2.8 & 23 & 5.6 & 41 & $2.0^{*}$ & 5.9 & 55 & $2.1^{* *}$ \\
\hline Sympt.\&lll-def. & & 10.4 & 158 & 7.4 & 104 & 13.8 & 189 & $1.9^{* *}$ & 15.5 & 252 & $2.1^{* *}$ \\
\hline \multirow{6}{*}{$\begin{array}{l}\text { EXTERNAL } \\
\text { CAUSES }\end{array}$} & & 61.5 & 1795 & 53.6 & 1608 & 56.2 & 1451 & 1.0 & 79.0 & 2267 & $1.5^{* *}$ \\
\hline & Road Accident & 14.4 & 527 & 12.8 & 469 & 5.8 & 210 & $0.5^{* *}$ & 20.1 & 739 & $1.6^{* *}$ \\
\hline & $\begin{array}{l}\text { Non transp.acc. } \\
\text { (Poison/fall/envir.) }\end{array}$ & 15.8 & 358 & 12.8 & 304 & 18.6 & 403 & $1.5^{* *}$ & 20.8 & 450 & $1.6^{* *}$ \\
\hline & Suicide & 26.2 & 760 & 23.8 & 717 & 18.5 & 483 & $0.8^{* *}$ & 33.3 & 937 & $1.4^{* *}$ \\
\hline & Event of Undet.Intent & 2.9 & 85 & 2.5 & 71 & 9.3 & 254 & $3.7^{* *}$ & 1.9 & 54 & 0.8 \\
\hline & Miscell.oth.Ext.causes & 2.3 & 65 & 1.7 & 47 & 4.0 & 101 & $2.3^{* *}$ & 2.9 & 88 & $1.7^{* *}$ \\
\hline
\end{tabular}

Age-adjusted rates and Potential Years of Life Lost rates per 100.000.

*ICD Chapters with fewer than 200 cases a year are not displayed.

Significance levels of the $z$ test: ${ }^{\prime * * \prime}=p<0.01 ;{ }^{\prime * \prime}=p<0.05$; ' $n s^{\prime}=$ Not significant. 
Table 5 Regional Comparisons of Causes of Premature deaths in Females, Belgium 2008-2009

\begin{tabular}{|c|c|c|c|c|c|c|c|c|c|c|c|}
\hline $\begin{array}{l}\text { Causes of death } \\
\text { Grouped }\end{array}$ & Detailed Cause & $\begin{array}{c}\text { RATES } \\
\text { Belgium }\end{array}$ & $\begin{array}{c}\text { PYLL } \\
\text { Belgium }\end{array}$ & $\begin{array}{l}\text { RATES } \\
\text { Flanders }\end{array}$ & $\begin{array}{c}\text { PYLL } \\
\text { Flanders }\end{array}$ & $\begin{array}{c}\text { RATES } \\
\text { Brussels }\end{array}$ & $\begin{array}{c}\text { PYLL } \\
\text { Brussels }\end{array}$ & $\begin{array}{l}\text { Rates ratio } \\
\text { Bxl/Fla }\end{array}$ & $\begin{array}{c}\text { RATES } \\
\text { Wallonia }\end{array}$ & $\begin{array}{c}\text { PYLL } \\
\text { Wallonia }\end{array}$ & $\begin{array}{l}\text { Rates ratio } \\
\text { Wal/Fla }\end{array}$ \\
\hline ALL PREMATURE & & 251.2 & 3440 & 224.4 & 3078 & 275.8 & 3757 & $1.2^{* *}$ & 295.3 & 4019 & $1.3^{* *}$ \\
\hline $\begin{array}{l}\text { NATURAL } \\
\text { CAUSES }\end{array}$ & & 227.4 & 2834 & 203.2 & 2526 & 250.5 & 3137 & $1.2^{* *}$ & 267.0 & 3317 & $1.3^{* *}$ \\
\hline \multirow{12}{*}{$\begin{array}{l}\text { ALL } \\
\text { NEOPLASMS }\end{array}$} & & 111.0 & 1445 & 106.1 & 1398 & 120.0 & 1548 & $1.1^{* *}$ & 118.1 & 1506 & $1.1^{* *}$ \\
\hline & $\begin{array}{l}\text { Head, Neck \& } \\
\text { oesoph.Ca }\end{array}$ & 3.6 & 46 & 3.1 & 41 & 4.5 & 60 & 1.4 & 4.3 & 52 & $1.4^{*}$ \\
\hline & Stomach Ca & 2.2 & 30 & 2.0 & 30 & 2.3 & 38 & 1.1 & 2.4 & 27 & 1.2 \\
\hline & Colorectal Ca & 9.8 & 107 & 9.7 & 104 & 10.8 & 126 & 1.1 & 9.8 & 105 & 1.0 \\
\hline & Liver Ca & 2.4 & 23 & 2.1 & 20 & 3.3 & 37 & 1.6 & 2.7 & 25 & 1.2 \\
\hline & Pancreas Ca & 5.5 & 57 & 5.3 & 59 & 6.8 & 70 & 1.3 & 5.7 & 50 & 1.1 \\
\hline & Lung Ca & 19.7 & 248 & 17.0 & 211 & 23.3 & 287 & $1.4^{* *}$ & 23.9 & 306 & $1.4^{* *}$ \\
\hline & Breast Ca & 25.2 & 364 & 25.6 & 369 & 26.7 & 407 & 1.0 & 24.2 & 345 & 0.9 \\
\hline & Female genital org.ca & 13.4 & 171 & 13.2 & 174 & 14.3 & 185 & 1.1 & 13.5 & 162 & 1.0 \\
\hline & CNS Ca & 4.0 & 76 & 4.3 & 81 & 3.0 & 45 & $0.7^{*}$ & 3.7 & 76 & 0.9 \\
\hline & Hematol.Ca & 7.4 & 99 & 7.4 & 107 & 6.6 & 83 & 0.9 & 7.7 & 91 & 1.0 \\
\hline & Other Neoplasms & 17.7 & 223 & 16.4 & 202 & 18.4 & 209 & 1.1 & 20.2 & 266 & $1.2^{* *}$ \\
\hline \multirow{4}{*}{$\begin{array}{l}\text { CIRCULATORY } \\
\text { DISEASES }\end{array}$} & & 49.0 & 510 & 43.8 & 436 & 53.1 & 560 & $1.2^{* *}$ & 57.5 & 626 & $1.3^{* *}$ \\
\hline & Isc.Heart Dis. & 15.1 & 142 & 13.8 & 124 & 16.7 & 154 & 1.2 & 17.0 & 171 & $1.2^{* *}$ \\
\hline & Cerebrov.dis.\&HTA & 13.9 & 150 & 12.6 & 132 & 15.2 & 158 & 1.2 & 15.9 & 180 & $1.3^{* *}$ \\
\hline & Other Circ.Dis. & 20.0 & 218 & 17.4 & 180 & 21.1 & 249 & $1.2^{*}$ & 24.5 & 275 & $1.4^{* *}$ \\
\hline $\begin{array}{l}\text { ALL OTH.NAT. } \\
\text { CAUSES }\end{array}$ & & 67.5 & 879 & 53.3 & 691 & 77.4 & 1029 & $1.5^{* *}$ & 91.5 & 1184 & $1.7^{* *}$ \\
\hline $\begin{array}{l}\text { Inf.\&parasit. } \\
\text { diseases }\end{array}$ & & 5.9 & 86 & 4.2 & 61 & 8.2 & 134 & $1.9^{* *}$ & 8.5 & 118 & $2.0^{* *}$ \\
\hline Endocrine dis & & 6.0 & 77 & 4.2 & 65 & 8.3 & 102 & $2.0^{* *}$ & 8.9 & 94 & $2.1^{* *}$ \\
\hline $\begin{array}{l}\text { Mental\&Neurol } \\
\text { Dis. }\end{array}$ & & 14.5 & 184 & 12.4 & 148 & 15.2 & 196 & $1.2^{*}$ & 18.3 & 246 & $1.5^{* *}$ \\
\hline \multirow[t]{2}{*}{ Resp.Syst.dis. } & & 16.8 & 173 & 13.7 & 137 & 18.4 & 178 & $1.3^{* *}$ & 22.4 & 238 & $1.6^{* *}$ \\
\hline & Chron.Obs.Pulm.Dis. & 9.2 & 89 & 7.1 & 67 & 9.7 & 97 & $1.4^{*}$ & 13.0 & 127 & $1.8^{* *}$ \\
\hline \multirow[t]{2}{*}{ Digest.Syst.Dis. } & & 12.7 & 185 & 10.0 & 135 & 13.0 & 187 & $1.3^{*}$ & 17.7 & 274 & $1.8^{* *}$ \\
\hline & Chronic Liver Dis. & 6.0 & 103 & 4.0 & 66 & 7.1 & 119 & $1.8^{* *}$ & 9.2 & 167 & $2.3^{* *}$ \\
\hline $\begin{array}{l}\text { Genito-urin.Syst. } \\
\text { Dis }\end{array}$ & & 2.8 & 26 & 1.9 & 17 & 3.7 & 46 & $1.9^{*}$ & 4.2 & 37 & $2.2^{* *}$ \\
\hline Sympt.\&lll-def. & & 4.6 & 63 & 3.2 & 41 & 5.4 & 71 & $1.7^{*}$ & 7.0 & 101 & $2.2^{* *}$ \\
\hline \multirow{6}{*}{$\begin{array}{l}\text { EXTERNAL } \\
\text { CAUSES }\end{array}$} & & 23.8 & 606 & 21.2 & 552 & 25.3 & 620 & $1.2^{*}$ & 28.3 & 702 & $1.3^{* *}$ \\
\hline & Road Accident & 3.9 & 133 & 3.5 & 123 & 2.2 & 63 & $0.6^{*}$ & 5.3 & 177 & $1.5^{* *}$ \\
\hline & $\begin{array}{l}\text { Non transp.acc. } \\
\text { (Poison/fall/envir.) }\end{array}$ & 7.1 & 130 & 5.6 & 98 & 8.2 & 172 & $1.5^{*}$ & 9.6 & 171 & $1.7^{* *}$ \\
\hline & suicide & 9.8 & 266 & 9.4 & 265 & 7.9 & 208 & 0.8 & 11.0 & 290 & $1.2^{*}$ \\
\hline & Event of Undet.Intent & 1.4 & 37 & 1.1 & 28 & 4.7 & 115 & $4.2^{* *}$ & 1.0 & 28 & 0.9 \\
\hline & Miscell.oth.Ext.causes & 1.5 & 39 & 1.5 & 37 & 2.3 & 61 & 1.5 & 1.3 & 36 & 0.8 \\
\hline
\end{tabular}

Age-adjusted rates and Potential Years of Life Lost rates per 100.000. 
Table 6 Top 20 causes of deaths in males by region, Belgium 2008-2009

\begin{tabular}{|c|c|c|c|c|}
\hline $\begin{array}{l}\text { Rank based on } \\
\text { PYLL }\end{array}$ & $\begin{array}{l}\text { Cause of death } \\
\text { Belgium }\end{array}$ & $\begin{array}{l}\text { Cause of death } \\
\text { Flanders }\end{array}$ & $\begin{array}{l}\text { Cause of death } \\
\text { Brussels }\end{array}$ & $\begin{array}{l}\text { Cause of death } \\
\text { Wallonia }\end{array}$ \\
\hline 1 & Suicide & Suicide & Lung $\mathrm{Ca}$ & Suicide \\
\hline 2 & Lung Ca & Lung Ca & Isc.Heart Dis. & Lung Ca \\
\hline 3 & Road Accident & Road Accident & Suicide & Road Accident \\
\hline 4 & Isc.Heart Dis. & Isc.Heart Dis. & Other Circ.Dis. & Isc.Heart Dis. \\
\hline 5 & Other Circ.Dis. & Other Circ.Dis. & Digest.Syst.Dis. & Mental\&Neurol Dis. \\
\hline 6 & $\begin{array}{l}\text { Non transp.acc. } \\
\text { (Poison/fall/envir.) }\end{array}$ & $\begin{array}{l}\text { Non transp.acc. } \\
\text { (Poison/fall/envir.) }\end{array}$ & $\begin{array}{l}\text { Non transp.acc. } \\
\text { (Poison/fall/envir.) }\end{array}$ & Digest.Syst.Dis. \\
\hline 7 & Digest.Syst.Dis. & Digest.Syst.Dis. & Resp.Syst.dis. & Other Circ.Dis. \\
\hline 8 & Mental\&Neurol Dis. & Mental\&Neurol Dis. & Mental\&Neurol Dis. & Resp.Syst.dis. \\
\hline 9 & Resp.Syst.dis. & Resp.Syst.dis. & Event of Undet.Intent & $\begin{array}{l}\text { Non transp.acc. } \\
\text { (Poison/fall/envir.) }\end{array}$ \\
\hline 10 & Head,neck\& oesoph.Ca & Head,neck\& oesoph.Ca & Cerebrov.dis.\&HTA & Head,neck\& oesoph.Ca \\
\hline 11 & Cerebrov.dis.\&HTA & Cerebrov.dis.\&HTA & Head,neck\& oesoph.Ca & Sympt\&lll-def. \\
\hline 12 & Sympt\&lll-def. & Colorectal Ca & Road Accident & Cerebrov.dis.\&HTA \\
\hline 13 & Colorectal Ca & Hematol.Ca & Sympt\&lll-def. & Inf.\&parasit.diseases \\
\hline 14 & Hematol.Ca & CNS Ca & Inf.\&parasit.diseases & Colorectal Ca \\
\hline 15 & Endocrine dis & Sympt\&lll-def. & Colorectal Ca & Endocrine dis \\
\hline 16 & Inf.\&parasit.diseases & Endocrine dis & Endocrine dis & Hematol.Ca \\
\hline 17 & CNS Ca & Pancreas Ca & Hematol.Ca & Pancreas Ca \\
\hline 18 & Pancreas Ca & Inf.\&parasit.diseases & Pancreas Ca & CNS Ca \\
\hline 19 & Event of Undet.Intent & Event of Undet.Intent & Miscell.oth.Ext.causes & Miscell.oth.Ext.causes \\
\hline 20 & Miscell.oth.Ext.causes & Male genital org.Ca & Stomach $\mathrm{Ca}$ & Liver Ca \\
\hline
\end{tabular}

Within the external causes, road accident decreased by $44 \%$; suicide rate slightly decreased $(-11 \%)$, while the non-transport accident increased (by $10 \%$ ).

Changes over time are nearly of the same magnitude when expressed in PYLL or in mortality rates. However, for some conditions, the decrease in PYLL was faster than the decrease in rates, meaning that the mortality reduction concerned more younger than older people: this was the case for head \& neck and haematological cancers, non-transport accident and event of undetermined intent.

In women also, the overall premature death rates has steadily declined over the 4 time periods, reaching a $22 \%$ decrease between the 1 st and the 4th period. Rates resulting of natural or external causes declined similarly.

Within the natural causes, circulatory diseases deaths rates decreased much faster $(-44.9 \%)$ than those due to neoplasms $(-13.7 \%)$ or to the other natural causes $(-9.8 \%)$. Among deaths resulting from cancer, the decrease was the fastest for stomach $(-39.2 \%)$. Colorectal, breast, haematological, gynaecological and CNS decreased by $20-30 \%$. Pancreas cancer death rates remain stable. The most worrying feature is the $50.2 \%$ increase in the lung cancer death rate. As in men, liver cancer increased by $16 \%$.
Within the external causes, road accident decreased by $51.9 \%$; the slight changes in suicide rate $(-9 \%)$ or in non-transport accident (by 5\%) are not significant.

PYLL decreased faster than mortality rates from breast, gynaecological, haematological, liver and lung cancers, and non-transport accident deaths, suggesting a more favourable evolution in young women. The reverse was seen for stomach cancer deaths.

\section{Discussion}

Recently published data on causes of death were used to analyse the burden, the ranking, the regional disparities and the 15 years evolution of premature mortality in Belgium, filling in a 12 years gap in Belgian figures. The addition of the PYLL indicator to the classical ageadjusted mortality rates completes the picture by weighting the burden of each condition with the loss of quantity of life. Ranking the causes of premature death based on PYLL helps setting up priorities for policymakers. However, since ranks are ordinal variables, they provide no quantitative information over the burden of the causes; therefore, one should always consider the value of the PYLL (or of the rates) for a specific cause, above its ranking when setting up priorities or to make regional comparison. 
Table 7 Top 20 causes of deaths in females by region, Belgium 2008-2009

\begin{tabular}{|c|c|c|c|c|}
\hline Rank based on PYLL & $\begin{array}{l}\text { Cause of death } \\
\text { Belgium }\end{array}$ & $\begin{array}{l}\text { Cause of death } \\
\text { Flanders }\end{array}$ & $\begin{array}{l}\text { Cause of death } \\
\text { Brussels }\end{array}$ & $\begin{array}{l}\text { Cause of death } \\
\text { Wallonia }\end{array}$ \\
\hline 1 & Breast Ca & Breast Ca & Breast $\mathrm{Ca}$ & Breast Ca \\
\hline 2 & Suicide & Suicide & Lung Ca & Lung Ca \\
\hline 3 & Lung Ca & Lung $\mathrm{Ca}$ & Other Circ.Dis. & Suicide \\
\hline 4 & Other Circ.Dis. & Other Circ.Dis. & Suicide & Other Circ.Dis. \\
\hline 5 & Digest.Syst.Dis. & Female genital org.ca & Mental\&Neurol Dis. & Digest.Syst.Dis. \\
\hline 6 & Mental\&Neurol Dis. & Mental\&Neurol Dis. & Digest.Syst.Dis. & Mental\&Neurol Dis. \\
\hline 7 & Resp.Syst.dis. & Resp.Syst.dis. & Female genital org.ca & Resp.Syst.dis. \\
\hline 8 & Female genital org.ca & Digest.Syst.Dis. & Resp.Syst.dis. & Cerebrov.dis.\&HTA \\
\hline 9 & Cerebrov.dis.\&HTA & Cerebrov.dis.\&HTA & $\begin{array}{l}\text { Non transp.acc. } \\
\text { (Poison/fall/envir.) }\end{array}$ & Road Accident \\
\hline 10 & Isc.Heart Dis. & Isc.Heart Dis. & Cerebrov.dis.\&HTA & $\begin{array}{l}\text { Non transp.acc. } \\
\text { (Poison/fall/envir.) }\end{array}$ \\
\hline 11 & Road Accident & Road Accident & Isc.Heart Dis. & Isc.Heart Dis. \\
\hline 12 & $\begin{array}{l}\text { Non transp.acc. } \\
\text { (Poison/fall/envir.) }\end{array}$ & Hematol.Ca & Inf.\&parasit.diseases & Chronic Liver Dis. \\
\hline 13 & Colorectal Ca & Colorectal Ca & Colorectal Ca & Female genital org.ca \\
\hline 14 & Chronic Liver Dis. & $\begin{array}{l}\text { Non transp.acc. } \\
\text { (Poison/fall/envir.) }\end{array}$ & Chronic Liver Dis. & Chron.Obs.Pulm.Dis. \\
\hline 15 & Hematol.Ca & CNS Ca & Event of Undet.Intent & Inf.\&parasit.diseases \\
\hline 16 & Chron.Obs.Pulm.Dis. & Chron.Obs.Pulm.Dis. & Endocrine dis & Colorectal Ca \\
\hline 17 & Inf.\&parasit.diseases & Chronic Liver Dis. & Chron.Obs.Pulm.Dis. & Sympt\&lll-def. \\
\hline 18 & Endocrine dis & Endocrine dis & Hematol.Ca & Endocrine dis \\
\hline 19 & CNS Ca & Inf.\&parasit.diseases & Sympt\&ll-def. & Hematol.Ca \\
\hline 20 & Sympt\&lll-def. & Pancreas Ca & Pancreas Ca & CNS Ca \\
\hline
\end{tabular}

There are well-known shortcomings with mortality data: they do not capture morbidity nor quality of life aspects, they rely on death certificate data of which quality is questionable for certain age groups and certain causes, they are coded using a classification which evolves over time. However the advantages of vital statistics are evident: they are routinely available, exhaustive, and they provide information to support health policy. They are of particular interest for planning and evaluating interventions aiming to reduce premature mortality.

\section{Quality of death registration data}

Aelvoet and all $[20,21]$ investigated the quality of the coding of death certificates data in Belgium until 1997. The conclusion was that the quality had appreciably improved since 1993, when the responsibility of the coding became more centralized and was assigned to the regional Health Agencies (currently two: one is coding for Flanders and Brussels, the second is coding for Wallonia). Caution was however recommended for analysing long-time trends and studying older age groups. By restricting the present analysis to data from 1993 to 2009 and by limiting the scope to premature deaths (1-74 years), the more questionable time periods and age groups were avoided.

Despite coordination meetings between the regional and national levels aiming to minimize the variability in regional practices, the coding of death certificates in 2 regional Health Agencies can still result in differences in the implementation of ICD rules. This issue will be discussed along with the interpretation of regional disparities. In 1998 a new model of death certificate was introduced in Belgium: it is conform to the WHO model and is suitable to be filled in by the certifying physician. Hence, less coding errors are to be expected after the running-in period.

The certification of the cause of death by the physician represents a cornerstone in the production of mortality statistics, it is thus important that it achieves a sufficient quality level. It was not the purpose of the present study to focus on the quality of the certification, so the quality evaluation was limited to the calculation of the percentage of imprecise codes, as defined by the WHO [22]. This latter is still representing $12 \%$ in $2008-2009$ (8\% for the age groups $<75$ years). As a consequence, efforts should still be made to improve the quality of the deaths 
Table 8 First 5 causes of deaths by age groups and region in males, Belgium 2008-2009

\begin{tabular}{|c|c|c|c|c|c|c|c|c|}
\hline Age range & $\begin{array}{l}\text { Cause of death } \\
\text { Belgium }\end{array}$ & Rate & $\begin{array}{l}\text { Cause of death } \\
\text { Flanders }\end{array}$ & Rate & $\begin{array}{l}\text { Cause of death } \\
\text { Brussels }\end{array}$ & Rate & $\begin{array}{l}\text { Cause of death } \\
\text { Wallonia }\end{array}$ & Rate \\
\hline $1-39$ yrs & Suicide & 16.4 & Suicide & 16.8 & Suicide & 10.4 & Road Accident & 20.8 \\
\hline $1-39$ yrs & Road Accident & 15.3 & Road Accident & 13.9 & $\begin{array}{l}\text { Non transp.acc. } \\
\text { (Poison/fall/envir.) }\end{array}$ & 7.3 & Suicide & 17.9 \\
\hline $1-39$ yrs & $\begin{array}{l}\text { Non transp.acc. } \\
\text { (Poison/fall/envir.) }\end{array}$ & 6.8 & $\begin{array}{l}\text { Non transp.acc. } \\
\text { (Poison/fall/envir.) }\end{array}$ & 6.7 & Road Accident & 6.0 & $\begin{array}{l}\text { Non transp.acc. } \\
\text { (Poison/fall/envir.) }\end{array}$ & 7.0 \\
\hline $1-39$ yrs & Mental\&Neurol Dis. & 4.1 & Mental\&Neurol Dis. & 2.9 & Event of Undet.Intent & 5.4 & Mental\&Neurol Dis. & 6.8 \\
\hline $1-39 y r s$ & Other Circ.Dis. & 2.6 & Other Circ.Dis. & 2.3 & Other Circ.Dis. & 2.4 & Other Circ.Dis. & 3.2 \\
\hline $40-59$ yrs & Lung Ca & 53.2 & Lung Ca & 46.3 & Lung Ca & 49.8 & Lung Ca & 67.0 \\
\hline $40-59$ yrs & Isc.Heart Dis. & 44.6 & Isc.Heart Dis. & 35.4 & Isc.Heart Dis. & 47.9 & Isc.Heart Dis. & 60.9 \\
\hline $40-59$ yrs & Suicide & 40.9 & Suicide & 34.2 & Digest.Syst.Dis. & 41.5 & Suicide & 56.9 \\
\hline $40-59$ yrs & Digest.Syst.Dis. & 35.7 & Other Circ.Dis. & 30.4 & Other Circ.Dis. & 34.3 & Digest.Syst.Dis. & 55.5 \\
\hline $40-59$ yrs & Other Circ.Dis. & 33.9 & Digest.Syst.Dis. & 24.2 & Suicide & 27.5 & Mental\&Neurol Dis. & 40.9 \\
\hline $60-74$ yrs & Lung Ca & 286.5 & Lung Ca & 277.4 & Lung Ca & 255.8 & Lung Ca & 312.6 \\
\hline $60-74$ yrs & Isc.Heart Dis. & 210.7 & Isc.Heart Dis. & 194.5 & Isc.Heart Dis. & 238.1 & Isc.Heart Dis. & 237.0 \\
\hline $60-74$ yrs & Other Circ.Dis. & 184.1 & Other Circ.Dis. & 177.0 & Resp.Syst.dis. & 215.7 & Resp.Syst.dis. & 230.7 \\
\hline 60- 74 yrs & Resp.Syst.dis. & 178.9 & Resp.Syst.dis. & 149.1 & Other Circ.Dis. & 173.7 & Other Circ.Dis. & 201.2 \\
\hline 60- 74 yrs & Cerebrov.dis.\&HTA & 94.1 & Cerebrov.dis.\&HTA & 84.9 & Digest.Syst.Dis. & 96.2 & Cerebrov.dis.\&HTA & 114.3 \\
\hline
\end{tabular}

Age specific rate per 100.000 .

certificates by insuring continuous training in death certification for the medical doctors and large dissemination of certification guidelines

Moreover, regional differences in certification might induce differences in observed rates, For instance, the statement that the category "events of undefined intend" represents a higher rates in Brussels than in Flanders may be considered to be at least partly due to regional difference in the certification rather than in coding procedures, since the death certificates of Flanders and Brussels are coded by the same Regional Authority.

The present study covers 2 versions of the ICD $[9,10]$, ICD-10 being implemented in 1998 in Belgium. Janssen and all [23] examined the effect of coding changes within ICD revisions. They evaluated the proportion of discontinuity for the ICD_9 to ICD_10 change to be lower than the former changes, namely $4.6 \%$, and affecting more specifically dementia, other circulatory diseases, symptoms and ill-defined conditions, other diseases (than those specifically studied), and falls. Specific cancers deaths were hardly affected by those changes.

After evaluation of the data used in the present study, it was decided not to publish the 1998-9 cause specific results, except for neoplasms, because of instability in coding practices. We noticed a substantial improvement after year 2000, partly due to the use of automated coding programs. During the analysis inconsistencies were detected in time series and in regional patterns of mental and neurological diseases (data not shown). This is due to the fact that the codes used for dementia (mainly
F01, F03 and G30), are dispersed across 2 different chapters of the ICD. The choice of a specific dementia code relies both on the diagnosis reported (by the physician who completes the death certificate) and on the coding rules of the Regional Agency, and has some impact on the death rate for each of those disease groups. The specific study of dementia pattern was out of the scope of this paper, so that it was decided to pool the chapters about mental and neurological diseases together.

\section{The PYLL indicator}

The ranking of the conditions depends on the choice of the indicator. The PYLL indicator emphasizes causes of death occurring at younger ages (mainly external causes) and is generally considered more appropriate to study the burden of premature death and to help setting up priorities. Depending on the focus of the study, various age limits have been used to calculate the PYLL indicator $[1-5,24]$, leading to different rankings of the causes of death. For instance studies focusing on productivity losses only include productive ages, mainly 20-65 years, while in studies of premature deaths the upper cut-off ranges from 65 to the age corresponding to life expectancy, with inclusion or not of infant deaths.

In the current study the upper cut-off was set at 75 years for two reasons: reported conditions for deaths occurring after 75 years are generally less reliable because of more frequent competing causes of death in older people; moreover the choice of a 75 year upper- 
Table 9 First 5 causes of deaths by age groups and region in females, Belgium 2008-2009

\begin{tabular}{|c|c|c|c|c|c|c|c|c|}
\hline Age range & $\begin{array}{l}\text { Cause of death } \\
\text { Belgium }\end{array}$ & Rate & $\begin{array}{l}\text { Cause of death } \\
\text { Flanders }\end{array}$ & Rate & $\begin{array}{l}\text { Cause of death } \\
\text { Brussels }\end{array}$ & Rate & $\begin{array}{l}\text { Cause of death } \\
\text { Wallonia }\end{array}$ & Rate \\
\hline $1-39$ yrs & Suicide & 5.3 & Suicide & 5.8 & Suicide & 3.7 & Suicide & 5.2 \\
\hline $1-39$ yrs & Road Accident & 3.6 & Road Accident & 3.3 & $\begin{array}{l}\text { Non transp.acc. } \\
\text { (Poison/fall/envir.) }\end{array}$ & 2.4 & Road Accident & 4.8 \\
\hline $1-39$ yrs & Mental\&Neurol Dis. & 1.8 & Breast Ca & 1.6 & Event of Undet.Intent & 2.0 & Mental\&Neurol Dis. & 2.9 \\
\hline $1-39$ yrs & $\begin{array}{l}\text { Non transp.acc. } \\
\text { (Poison/fall/envir.) }\end{array}$ & 1.7 & Hematol.Ca & 1.5 & Breast Ca & 1.7 & $\begin{array}{l}\text { Non transp.acc. } \\
\text { (Poison/fall/envir.) }\end{array}$ & 2.4 \\
\hline $1-39$ yrs & Breast Ca & 1.6 & Mental\&Neurol Dis. & 1.2 & Mental\&Neurol Dis. & 1.7 & Other Circ.Dis. & 1.7 \\
\hline $40-59$ yrs & Breast Ca & 35.3 & Breast Ca & 34.7 & Breast Ca & 41.3 & Breast Ca & 34.6 \\
\hline $40-59$ yrs & Lung Ca & 25.4 & Lung Ca & 21.9 & Lung Ca & 26.1 & Lung Ca & 31.5 \\
\hline $40-59$ yrs & Digest.Syst.Dis. & 17.9 & Female genital org.ca & 16.0 & Other Circ.Dis. & 19.3 & Digest.Syst.Dis. & 28.2 \\
\hline $40-59$ yrs & Other Circ.Dis. & 16.5 & Suicide & 14.1 & Female genital org.ca & 15.9 & Other Circ.Dis. & 21.8 \\
\hline $40-59$ yrs & Suicide & 15.7 & Other Circ.Dis. & 13.2 & Digest.Syst.Dis. & 15.5 & Suicide & 19.5 \\
\hline $60-74$ yrs & Breast Ca & 90.8 & Breast Ca & 94.0 & Lung Ca & 97.0 & Other Circ.Dis. & 106.8 \\
\hline $60-74$ yrs & Other Circ.Dis. & 90.5 & Other Circ.Dis. & 81.9 & Breast Ca & 90.0 & Resp.Syst.dis. & 101.7 \\
\hline $60-74$ yrs & Resp.Syst.dis. & 79.3 & Isc.Heart Dis. & 68.5 & Other Circ.Dis. & 90.0 & Lung Ca & 93.6 \\
\hline $60-74$ yrs & Lung Ca & 78.2 & Lung Ca & 67.6 & Resp.Syst.dis. & 87.7 & Breast Ca & 85.1 \\
\hline 60- 74 yrs & Isc.Heart Dis. & 72.8 & Resp.Syst.dis. & 66.4 & Isc.Heart Dis. & 81.6 & Isc.Heart Dis. & 78.7 \\
\hline
\end{tabular}

Age specific rate per 100.000 .

limit is consistent with the recent definition of avoidable mortality [25].

The inclusion of infant deaths is also a matter of debate. They have been excluded from the present study, as recommended by Romeder [5], because infant mortality is due to causes that are very specific to this age group. Moreover, the weight given to infant deaths account for almost 75 years in the computed PYLL, which is probably an overestimation of the societal weight of those deaths in comparison of a death occurring in young adulthood. Finally, more appropriate indicators exist that specifically address death in this particular age group.

\section{Interpretation of findings}

Compared to the other EU15 countries, overall premature deaths rates and PYLL are quite high in Belgium. This comparison with similar countries highlights potential gains in life expectancy.

The male to female premature mortality ratio [2] is much higher than the one observed in all age mortality (1.6), suggesting that premature mortality contributes more to the gender difference in life expectancy than mortality at older ages.

Among men, the leading causes of premature deaths ranked according to PYLL are suicide, lung cancer, road accident, ischemic heart disease and other circulatory disease. Among women, breast cancer still occupies the top position, followed by suicide, lung cancer, other circulatory diseases, and digestive diseases (more than half of this latter being due to chronic liver disease). It is noteworthy that almost all of those causes are also considered as "avoidable death", meaning sensitive either to the health system or to public health interventions. There is room for improvement by further reducing those avoidable deaths.

\section{Regional differences}

The ranking of causes of deaths is roughly the same for Flanders and Wallonia, but differs somehow in Brussels, where road accidents rank much lower than in the other regions; suicide in men also occupies a lower place than in the other regions. Regional disparities in overall and cause specific mortality for all ages $[6,9,26,27]$, in premature mortality [8] and in avoidable mortality [28,29] have been documented in the past (until 1997). Since World War II, Wallonia always experienced a clear disadvantage in male mortality, mostly due to deaths related to circulatory diseases, neoplasms, respiratory diseases, chronic liver diseases, external causes and infectious diseases. At the contrary to our finding, in the older studies, stomach cancers were higher in Flanders than in the two other regions [7] and most of the other natural causes didn't show significant differences.

The present work updates, at the hand of the newly available vital statistics, the information about the regional disparities in overall and cause specific premature mortality. An important Walloon excess in male overall premature mortality as compared to the Flemish rates (Rate ratio: 1.4) is still observed, for the same specific causes as in previous studies. It is noteworthy that the 
Table 10 Evolution of premature deaths in males by main causes in Belgium, across 4 time-period, 1993-2009

\begin{tabular}{|c|c|c|c|c|c|c|c|c|c|c|c|}
\hline Cause of death & $\begin{array}{c}\text { RATES } \\
1993-1994\end{array}$ & $\begin{array}{c}\text { RATES } \\
1998-1999^{\$}\end{array}$ & $\begin{array}{c}\text { RATES } \\
2003-2004\end{array}$ & $\begin{array}{c}\text { RATES } \\
2008-2009\end{array}$ & $\begin{array}{c}15 \text { years change } \\
\text { RATES }\end{array}$ & $\begin{array}{c}\text { PYLL } \\
1993-1994\end{array}$ & $\begin{array}{c}\text { PYLL } \\
1998-1999\end{array}$ & $\begin{array}{c}\text { PYLL } \\
2003-2004\end{array}$ & $\begin{array}{c}\text { PYLL } \\
2008-2009\end{array}$ & $\begin{array}{c}15 \text { years change } \\
\text { PYLL }\end{array}$ & $\begin{array}{l}\text { Sign level change } \\
\text { in rates }\end{array}$ \\
\hline ALL PREMATURE & 661.9 & 601.6 & 522.0 & 466.3 & $-29.6 \%$ & 8834 & 8020 & 7109 & 6410 & $-27.4 \%$ & $\overline{* *}$ \\
\hline NATURAL CAUSES & 584.2 & . & 456.9 & 404.7 & $-30.7 \%$ & 6375 & . & 5111 & 4615 & $-27.6 \%$ & $* *$ \\
\hline ALL NEOPLASMS & 238.6 & 216.7 & 190.3 & 175.1 & $-26.6 \%$ & 2610 & 2343 & 2097 & 1914 & $-26.7 \%$ & $* *$ \\
\hline Head,Neck \& oesoph.Ca & 21.0 & 19.9 & 18.3 & 16.6 & $-20.8 \%$ & 317 & 288 & 254 & 219 & $-30.8 \%$ & $* *$ \\
\hline Stomach Ca & 9.8 & 7.8 & 6.3 & 5.1 & $-48.4 \%$ & 97 & 81 & 72 & 56 & $-42.4 \%$ & $* *$ \\
\hline Colorectal Ca & 20.4 & 19.4 & 16.4 & 15.6 & $-23.6 \%$ & 197 & 188 & 154 & 152 & $-22.8 \%$ & $* *$ \\
\hline Liver Ca & 5.1 & 5.1 & 5.4 & 5.9 & $16.0 \%$ & 54 & 53 & 58 & 64 & $18.9 \%$ & * \\
\hline Pancreas Ca & 9.3 & 9.3 & 8.7 & 9.0 & $-3.0 \%$ & 101 & 93 & 94 & 92 & $-9.0 \%$ & ns \\
\hline Lung Ca & 94.5 & 81.1 & 67.5 & 61.9 & $-34.6 \%$ & 944 & 803 & 682 & 630 & $-33.3 \%$ & $* *$ \\
\hline Male genital org.Ca & 14.7 & 13.0 & 10.0 & 8.8 & $-40.4 \%$ & 97 & 86 & 67 & 65 & $-33.3 \%$ & $* *$ \\
\hline CNS Ca & 7.4 & 6.7 & 5.5 & 5.9 & $-20.3 \%$ & 133 & 115 & 105 & 106 & $-20.3 \%$ & $* *$ \\
\hline Hematol.Ca & 15.6 & 13.9 & 12.7 & 11.5 & $-25.9 \%$ & 214 & 180 & 154 & 138 & $-35.4 \%$ & $* *$ \\
\hline Other Neoplasms & 40.8 & 40.7 & 39.5 & 34.9 & $-14.5 \%$ & 456 & 457 & 458 & 391 & $-14.3 \%$ & $* *$ \\
\hline CIRCULATORY DISEASES & 193.7 & . & 136.4 & 109.6 & $-43.4 \%$ & 1893 & . & 1400 & 1176 & $-37.9 \%$ & $* *$ \\
\hline ALL OTH.NAT.CAUSES & 151.9 & . & 130.3 & 120.0 & $-21.0 \%$ & 1871 & . & 1614 & 1525 & $-18.5 \%$ & $* *$ \\
\hline Chron.Obs.Pulm.Dis. & 38.1 & . & 25.0 & 21.5 & $-43.6 \%$ & 288 & . & 188 & 170 & $-41.2 \%$ & $* *$ \\
\hline Chronic Liver Dis. & 14.8 & . & 13.0 & 13.5 & $-8.9 \%$ & 267 & . & 224 & 217 & $-18.7 \%$ & * \\
\hline $\begin{array}{l}\text { Remaining Other Natural } \\
\text { Causes }\end{array}$ & 89.1 & & 81.2 & 76.3 & $-14.4 \%$ & 1119 & & 1056 & 1022 & $-8.7 \%$ & $* *$ \\
\hline EXTERNAL CAUSES & 77.7 & . & 65.0 & 61.5 & $-20.8 \%$ & 2459 & . & 1997 & 1795 & $-27.0 \%$ & $* *$ \\
\hline Road Accident & 25.7 & & 17.5 & 14.4 & $-44.0 \%$ & 966 & & 664 & 527 & $-45.5 \%$ & $* *$ \\
\hline $\begin{array}{l}\text { Non transp.acc.(Poison/fall/ } \\
\text { envir.) }\end{array}$ & 14.4 & . & 14.8 & 15.8 & $9.7 \%$ & 396 & . & 350 & 358 & $-9.6 \%$ & * \\
\hline Suicide & 29.5 & & 28.1 & 26.2 & $-11.4 \%$ & 871 & & 839 & 760 & $-12.7 \%$ & $* *$ \\
\hline Event of Undet.Intent & 3.1 & . & 2.0 & 2.9 & $-3.6 \%$ & 95 & . & 65 & 85 & $-11.0 \%$ & ns \\
\hline Miscell.oth.Ext.causes & 5.0 & & 2.4 & 2.3 & $-54.6 \%$ & 131 & & 76 & 65 & $-50.2 \%$ & $* *$ \\
\hline
\end{tabular}

Age-adjusted rates and age-adjusted PYLL rates per 100.000

15 years changes in rates and PYLL are displayed in bold.

\$.1998-1999 figures by detailed COD not shown for lack of reliability (except for neoplasms).

\$\$.Significance level of the change in Rates: $n$ s 'Not Significant' * ' $<0.05$ '** ' $<0.01$ '. 
Table 11 Evolution of premature deaths in females by main causes in Belgium, across 4 time-period, 1993-2009

\begin{tabular}{|c|c|c|c|c|c|c|c|c|c|c|c|}
\hline Cause of death & $\begin{array}{c}\text { RATES 1993- } \\
1994\end{array}$ & $\begin{array}{c}\text { RATES 1998- } \\
1999^{5}\end{array}$ & $\begin{array}{c}\text { RATES 2003- } \\
2004\end{array}$ & $\begin{array}{l}\text { RATES 2008- } \\
\quad 2009\end{array}$ & $\begin{array}{l}15 \text { years } \\
\text { change } \\
\text { RATES }\end{array}$ & $\begin{array}{c}\text { PYLL 1993- } \\
1994\end{array}$ & $\begin{array}{c}\text { PYLL 1998- } \\
1999\end{array}$ & $\begin{array}{c}\text { PYLL 2003- } \\
2004\end{array}$ & $\begin{array}{c}\text { PYLL 2008- } \\
2009\end{array}$ & $\begin{array}{c}15 \text { years } \\
\text { change } \\
\text { PYLL }\end{array}$ & $\begin{array}{c}\text { Sign level } \\
\text { change } \\
\text { in rates }\end{array}$ \\
\hline$\overline{\text { ALL PREMATURE }}$ & 322.2 & 296.9 & 267.2 & 251.2 & $-22.0 \%$ & 4358 & 4064 & 3630 & 3440 & $-21.1 \%$ & ** \\
\hline NATURAL CAUSES & 292.1 & . & 243.0 & 227.4 & $-22.2 \%$ & 3525 & & 2988 & 2834 & $-19.6 \%$ & $* *$ \\
\hline ALL NEOPLASMS & 128.5 & 121.3 & 111.3 & 111.0 & $-13.7 \%$ & 1749 & 1626 & 1468 & 1445 & $-17.4 \%$ & $* *$ \\
\hline Head,Neck \& oesoph.Ca & 3.5 & 3.7 & 3.9 & 3.6 & $3.0 \%$ & 53 & 53 & 55 & 46 & $-13.2 \%$ & ns \\
\hline Stomach Ca & 3.6 & 3.1 & 2.3 & 2.2 & $-39.2 \%$ & 38 & 36 & 27 & 30 & $-21.6 \%$ & $* *$ \\
\hline Colorectal Ca & 13.5 & 12.0 & 10.2 & 9.8 & $-27.3 \%$ & 146 & 131 & 106 & 107 & $-27.1 \%$ & $* *$ \\
\hline Liver Ca & 2.1 & 2.5 & 2.2 & 2.4 & $16.2 \%$ & 25 & 24 & 24 & 23 & $-5.8 \%$ & ns \\
\hline Pancreas Ca & 5.7 & 6.3 & 5.4 & 5.5 & $-2.0 \%$ & 57 & 63 & 50 & 57 & $0.6 \%$ & ns \\
\hline Lung Ca & 13.1 & 13.9 & 15.9 & 19.7 & $50.2 \%$ & 175 & 183 & 210 & 248 & $41.9 \%$ & $* *$ \\
\hline Breast Ca & 33.5 & 31.6 & 26.9 & 25.2 & $-24.6 \%$ & 517 & 482 & 411 & 364 & $-29.5 \%$ & $* *$ \\
\hline Female genital org.ca & 17.3 & 14.9 & 13.7 & 13.4 & $-22.8 \%$ & 232 & 199 & 172 & 171 & $-26.3 \%$ & $* *$ \\
\hline CNS Ca & 5.6 & 4.7 & 4.0 & 4.0 & $-29.0 \%$ & 103 & 84 & 70 & 76 & $-26.1 \%$ & $* *$ \\
\hline Hematol.Ca & 9.8 & 8.6 & 8.0 & 7.4 & $-24.9 \%$ & 144 & 116 & 105 & 99 & $-31.0 \%$ & $* *$ \\
\hline Other Neoplasms & 20.9 & 19.9 & 18.8 & 17.7 & $-15.0 \%$ & 260 & 254 & 237 & 223 & $-14.2 \%$ & $* *$ \\
\hline CIRCULATORY DISEASES & 88.8 & . & 61.7 & 49.0 & $-44.9 \%$ & 799 & . & 617 & 510 & $-36.2 \%$ & $* *$ \\
\hline ALL OTH.NAT.CAUSES & 74.8 & . & 70.0 & 67.5 & $-9.8 \%$ & 977 & & 902 & 879 & $-10.0 \%$ & $* *$ \\
\hline Chron.Obs.Pulm.Dis. & 10.2 & & 9.3 & 9.2 & $-9.8 \%$ & 101 & . & 92 & 89 & $-11.7 \%$ & * \\
\hline Chronic Liver Dis. & 7.7 & & 6.0 & 6.0 & $-22.3 \%$ & 134 & & 107 & 103 & $-23.0 \%$ & $* *$ \\
\hline $\begin{array}{l}\text { Remaining Other Natural } \\
\text { Causes }\end{array}$ & 52.3 & . & 47.5 & 46.4 & $-11.2 \%$ & 657 & . & 601 & 601 & $-8.5 \%$ & $* *$ \\
\hline EXTERNAL CAUSES & 30.1 & . & 24.2 & 23.8 & $-20.9 \%$ & 832 & & 642 & 606 & $-27.2 \%$ & $* *$ \\
\hline Road Accident & 8.2 & . & 5.2 & 3.9 & $-51.9 \%$ & 282 & . & 174 & 133 & $-52.6 \%$ & $* *$ \\
\hline $\begin{array}{l}\text { Non transp.acc.(Poison/fall/ } \\
\text { envir.) }\end{array}$ & 6.8 & & 6.5 & 7.1 & $5.1 \%$ & 152 & & 118 & 130 & $-14.5 \%$ & ns \\
\hline Suicide & 10.7 & & 10.1 & 9.8 & $-9.2 \%$ & 287 & & 280 & 266 & $-7.5 \%$ & * \\
\hline Event of Undet.Intent & 1.4 & . & 0.7 & 1.4 & $0.6 \%$ & 38 & & 20 & 37 & $-3.3 \%$ & ns \\
\hline Miscell.oth.Ext.causes & 2.9 & . & 1.7 & 1.5 & $-47.4 \%$ & 73 & & 51 & 39 & $-45.7 \%$ & $* *$ \\
\hline
\end{tabular}

Age-adjusted rates and age-adjusted PYLL rates per 100.000.

15 years changes in rates and PYLL are displayed in bold.

\$.1998-1999 figures by detailed COD not shown for lack of reliability (except for neoplasms).

$\$$. Significance level of the change in Rates: $n s$ 'Not Significant' * ' $<0.05^{\prime * *}$ ' $<0.01$ '. 
Wallonia-to-Flanders rate ratio for premature death is much higher than the rate ratio for all ages mortality, which is only 1.2 in 2009 (source = spma https://s9xjb. wiv-isp.be/SASStoredProcess/guest?_program=/SPMA/ $\mathrm{SP} /$ allcaus). Again, those large regional disparities highlight the possible room for improvement. Such regional disparities could be explained by differences in the socio-economic context, in health policies and to some extent in health care practices, by environmental factors and cultural differences in health behaviour. The socio-economic context is worse in Wallonia than in Flanders; however, an analysis of the other factors and particularly an evaluation of the health policies could highlight some causes of the observed differencesthat could be vulnerable to policy interventions.

The present study also yielded some new findings, e.g. the former regional difference in stomach cancer mortality (with Flanders at a disadvantage) has totally disappeared, probably because of a change in the way of conserving food. Beside this, a $80-100 \%$ excess was observed in Wallonia as compared to Flanders for many natural causes (grouped into ICD chapter, namely mental and neurological, digestive, endocrine diseases). This had never been described before: at the contrary, Van Houte-Minet [6] explicitly mentioned that no significant regional difference was observed for those specific causes during the period 1970-72. Other previous studies did not specifically examine regional disparities for those causes.

Men in Brussels experience a premature mortality rate intermediate between the Flemish and Walloon rates, with a main Brussels-to-Flanders excess in the group of "other natural causes" $(+60 \%)$, then in the circulatory diseases $(+20 \%)$, and cancers $(10 \%)$. While no Brussels-to-Flanders difference is observed in the overall rate of external causes, large differences are observed in the specific external causes of deaths: for evident reasons, the road accidents rate is much lower than in the other regions. The suicide rate, even after the Jougla's correction, is lower in Brussels than in the other regions and no clear explanation for this could be found. It is noteworthy that the category of nontransport accident (in our classification: fall, poisoning and environmental accidents) is higher than in the other regions, which should be further explored: there are possibly more infrastructure problems in housing in big towns, however suicide declared as accidental poisoning cannot be excluded. The unexplained high rate of events of undetermined intent in Brussels requires caution in the interpretation of specified external causes.

Among women, the overall Wallonia to Flanders rate ratio for premature death is slightly less pronounced than in men $(R R=1.3)$. The $R R$ for cancer is only 1.1
( $10 \%$ excess); this is mainly attributable to smoking related cancers, namely head \& neck and lung cancers in which the Walloon excess reaches $40 \%$. Rate ratios in other causes show the same pattern as in men.

As it was the case for men, premature mortality rates for women in Brussels are in between those of Flanders and Wallonia, both for overall mortality and for natural cause mortality. For external causes, a similar pattern is observed as in men, with a lower rate of road accidents and suicide, and a higher rate of non-transport accident.

Although coding was processed in two separate health agencies, it is unlikely that this could explain much of the observed regional differences. The difference in overall mortality is anyway not related to different coding rules. Second, as the category "symptoms and ill-defined causes" represents a higher proportion of deaths in Wallonia (2.6\% in 2008-9) than in Flanders (1.7\%) and is almost the same as in Brussels (2.5\%), any reclassification of poorly into better-defined chapters would result in an increase of specific death rates, that would be larger in Wallonia than in Flanders, still accentuating the regional differences. Finally, differences at highly aggregated levels, such as head of ICD chapters, could hardly be due to variations in coding practices. This was only the case for mental and neurological diseases, a problem that was solved by pooling the categories.

Only a few of single causes have been studied in detail, namely the cancers, circulatory diseases, COPD, chronic liver diseases and external causes. The coding of cancer type is unlikely to be subject to regional differences; regional differences in COPD and chronic liver diseases deaths have since long been documented and explained by life styles and working environment differences. However, a regional coding bias is not excluded for specific circulatory diseases, so that it is preferable to only compare the pooled category of circulatory diseases.

The category external causes is also prone to create differences in coding and should be examined with caution. A clear regional bias is observed for the category "event of undetermined intent", which is much higher in Brussels. Technics of correcting for not-at-random missing values could be used to further explore the apparent differences in external deaths.

Measuring changes in the magnitude of the regional disparities over time was out of the scope of this study, and methodological differences between previous studies and this one ask for more in depth research.

\section{Evolution of the national rates over time}

Among men, a substantial decrease is observed in death rates due to road accidents, probably as a result of drastic measures in the road security policy. Regarding death related to tobacco use, a major decrease is observed in circulatory diseases, larger than the one observed for 
head \& neck and lung cancer. This discrepancy may suggest the influence of additional factors as changes in diet, prevention and medical treatment of hypertension. Despite the progress observed in the last decade, road accidents, lung cancers and circulatory diseases remain the main causes of potential years of life lost, and policymakers should pursue efforts to further reduce them.

The important decrease in stomach cancer likely results from a change in the methods of food preservation, leading to a reduction in salt consumption. The reduction in haematological and reproductive organ cancer death rates is possibly due to improvement of chemotherapy schemes.

Suicide has hardly declined and is nowadays the main cause of premature deaths in men at national level, when measured in PYLL; moreover, if the correction for underreporting is applied, suicide far exceeds all other single causes of death in men, except in Brussels.

The slight increase of liver cancer mortality should be further explored; this could result from a change in reporting, but increased viral hepatitis or alcohol consumption could also play a role.

The stability in non-transport accidents (fall, poisoning, environment) deaths should be checked, as it has been reported that the change in ICD version impact the way of coding for those specific causes [23].

Among women, the 15 years decrease in overall mortality is slightly lower than in men $(22 \%$ decrease in mortality rates). Already lower mortality rates are probably harder to improve. However, attention should be paid to the fact that the decrease in cancer rates $(-14 \%)$ is much slower than in circulatory diseases $(-45 \%)$, and slower than in men $(-27 \%)$. The $50 \%$ increase in lung cancer rates and the stability in head \& neck cancer rates are responsible for this slower evolution. The smoking behaviour of the women could potentially explain this evolution. As in men, the discrepancy between the evolution in lung cancer and in circulatory diseases mortality suggest that smoking is not the only factor influencing the latter rate.

\section{Conclusion}

An impressive improvement in premature mortality over the last 15 years has contributed to an increase in life expectancy. Our study shows that mortality declined for most causes, but the $50 \%$ increase in female lung cancer mortality is an alarming evolution. The comparison with EU15 countries proofs that there is room for improvement. This is even more the case for the Walloon region with distinct higher overall and cause specific mortality. Even if the less favourable socio-economic context in Wallonia certainly contributes to these differences, an in-depth evaluation of health policies and health system performance would give some insights to the roots of the regional disparities and contribute to improve the situation.

\section{Abbreviations}

PYLL: Potential years of life lost; WHO: World Health Organisation; Statistics Belgium: Directorate-general statistics Belgium; ICD: International classification of diseases; CNS Ca: 'Central nervous system cancer';

CPOD: Chronic pulmonary obstructive disease; Isc.Heart Dis.: Ischemic heart diseases'; Cerebrov.dis.\&HTA: 'Cerebrovascular disease and hypertension'; Sympt\&lll-def: Symptoms and ill-defined conditions; Non transp.acc. (Poison/fall/envir.): 'Non transport accidents (Poisoning, fall, environmental accidents).

\section{Competing interests}

The authors declare that they have no competing interests.

\section{Authors' contribution}

FR performed the statistical analysis, was involved in the interpretation of results and drafted the manuscript. JT was involved in the interpretation of results and the reviewing of the manuscript. PD was involved in the conceptual phase, interpretation of results and writing of the manuscript. All authors read and approved the final manuscript.

\section{Acknowledgements}

We thank Sabine Drieskens for her help in SAS programmation.

\section{Author details}

'Department Public Health and Statistics, Institute of Public Health, Brussels, Belgium. ${ }^{2}$ Interface Demography, Section Social Research, Free University of Brussels, Brussels, Belgium.

Received: 18 March 2014 Accepted: 1 July 2014

Published: 1 October 2014

\section{References}

1. Mackenbach J, Karanikolos M, Mckee M: Past and future health gains. In Successes and failures of health policies in Europe. Edited by Mackenbach J, Mckee M. Berkshire: Open University Press; 2013:286-98.

2. Center for Diseases Control: Premature mortality in the United States: public health issues in the use of years of potential life lost. MMWR Morb Mortal Wkly Rep 1986, 35(2 Suppl):1S-11S.

3. Perloff JD, LeBailly SA, Kletke PR, Budetti PP, Connelly JP: Premature death in the United States: years of life lost and health priorities. $J$ Public Health Policy 1984, 5(2):167-84.

4. Haenszel W: A standardized rate for mortality defined in units of lost years of life. Am J Public Health Nations Health 1950, 40(1):17-26.

5. Romeder JM, McWhinnie JR: Potential years of life lost between ages 1 and 70: An indicator of premature mortality for health planning. Int J Epidemiol 1977, 6:143-51

6. Van Houte-Minet $\mathrm{M}$, Wunsch G: La mortalité masculine aux âges adultes, un essai d'analyse régionale. Population et Famille 1978, 43:37-68.

7. Van Houte-Minet M, Wunsch G: La mortalité masculine aux âges adultes: causes et déterminants régionaux. Population et Famille 1978, 44(2):19-48.

8. Leveque A, Berghmans L, Dramaix M, Lagasse R: La mortalité prématurée en Belgique (Région Wallone) en 1986: les années potentielles de vie perdues comme indicateur d'importance et de tendance (1976-1986). Arch Public Health 1992, 50:141-57.

9. Puddu M, Drieskens S, Tafforeau J, de Roubaix J, Pirenne Y, Binon J, Diament M, Crapanzano L: Statistiques de Décès en Communauté Française 1995-1997. Brussels: CROSP; 2003.

10. WHO: International Classification of Diseases, injuries and causes of death, based on the 9th Revision Conference. Geneva: World Health Organisation; 1977.

11. WHO: International Statistical Classification of Diseases and Related Health Problems: 10th Revision. Geneva: World Health Organisation; 1994.

12. Moens GFG: The reliability of reported suicide mortality statistics: An experience from Belgium. Int J Epidemiol 1985, 14(2):272-5.

13. Jougla E, Pequignot F, Chappert J, Rossollin F, Le TA, Pavillon G: Quality of suicide mortality data. Rev Epidemiol Sante Publique 2002, 50(1):49-62.

14. Ohberg A, Lonnqvist J: Suicides hidden among undetermined deaths. Acta Psychiatrica Scandinavica 1998, 98(3):214-8.

15. De Spiegelaere M, Wauters I, Haelterman E: Le suicide en Région de Bruxelles-Capitale: Situation 1998-2000. Brussels: Observatoire de la santé et du social de Bruxelles- Capitale; 2003. 
16. Jensen OM, Parkin DM, Maclennan R, Muir CS, Skeet RG: Cancer registration: principles and methods. International Agency for Research on Cancer: Lyon; 1991.

17. Segi M, Fujisaku S, Kurihama M, Naray Y, Sasajima K: The age-adjusted death rates for malignant neoplasms in some selected sites in 23 countries in 1954-1955 and their geographical correlation. Tohoku J Exp Med 1960, 72:91-103.

18. Armitage P, Bery G: Statistical methods in medical research. Oxford: Blackwell Scientific Publications; 1987.

19. Smith P: Comparison between registries: age-standardized rates. In Cancer incidence in five continents, Volume V. Edited by Muir C, Waterhouse J, Mack T, Powell J, Whelan S. Lyon: IARC; 1987:790-5.

20. Aelvoet W: Gezondheidsindicatoren, 1994. Ministerie van de Vlaamse Gemeenschap: Brussel; 1996.

21. Aelvoet $W$, Molenberghs $G$, van Sprundel M: Mortality statistics in Belgium 1980-1997: quality of coding. Arch Public Health 2005, 63:127-48.

22. Mathers CD, Fat DM, Inoue M, Rao C, Lopez AD: Counting the dead and what they died from: an assessment of the global status of cause of death data. Bull World Health Organ 2005, 83(3):171-7.

23. Janssen F, Kunst AE: ICD coding changes and discontinuities in trends in cause-specific mortality in six European countries, 1950-99. Bull World Health Organ 2004, 82(12):904-13.

24. Gardner JW, Sanborn JS: Years of Potential Life Lost (YPLL). What Does it Measure? Epidemiol 1990, 1:322-9.

25. Nolte $E$, Mckee M: Measuring the health of nations: analysis of mortality amenable to health care. BMJ 2003, 327(7424):1129.

26. Dooghe D: Gedifferentieerd sterftebeeld: toepassing U. Yule method. Population et Famille 1965, 6:211-29.

27. Duchene J, Thiltges E: La mortalité des plus de 15 ans en Belqique: les disparités régionales en 1985-87, Espace, populations, sociétés; 1993:64-71.

28. Humblet PC, Lagasse R, Moens GFG, Wollast E, Van de Voorde H: La mortalité évitable en Belgique. Soc Sci Med 1987, 25:485-93.

29. Humblet $P C$, Lagasse $R$, Leveque A: Trends in Belgian premature avoidable deaths over a 20 year period. $J$ Epidemiol Community Health 2000, 54(9):687-91.

\section{Submit your next manuscript to BioMed Central and take full advantage of:}

- Convenient online submission

- Thorough peer review

- No space constraints or color figure charges

- Immediate publication on acceptance

- Inclusion in PubMed, CAS, Scopus and Google Scholar

- Research which is freely available for redistribution 